\title{
A TECHNIQUE FOR STUDYING THE MORPHOLOGY OF MAMMALIAN SPERMATOZOA WHICH ARE EOSINOPHILIC IN A DIFFERENTIAL 'LIVE/DEAD' STAIN
}

\author{
H. M. DOTT and G. C. FOSTER \\ Unit of Reproductive Physiology and Biochemistry, Animal Research Station, \\ 307 Huntingdon Road, Cambridge CB3 0JQ \\ (Received 6th Fanuary 1972, accepted 13th Fanuary 1972)
}

The purpose of a differential stain is to colour the cells concerned in such a way that two populations can be distinguished from each other and from the background. This is certainly true of staining mixtures used to differentiate 'live' and 'dead' mammalian spermatozoa. In practice, the distinction is between eosinophilic and non-eosinophilic cells with a secondary stain (e.g. nigrosin) which appears only to provide a background.

The degree of background staining can be varied from none to intense by varying the concentration of the secondary stain. At very low concentration, the non-eosinophilic spermatozoa tend to merge with the background, their outline is difficult to distinguish clearly and, consequently, they may be overlooked in a count. As the concentration of the background stain is increased, the noneosinophilic spermatozoa can be discerned clearly and easily but the outline of the stained spermatozoa becomes indistinct, particularly in the region of the acrosome.

A method has been developed which enables a 'live/dead' count and a detailed examination of morphology to be carried out on the same slide. The method has been successful with semen from all species whose spermatozoa are differentiated by a nigrosin and eosin stain. The semen was obtained by electroejaculation or artificial vagina or from the epididymis of slaughtered animals. The method can also be used with deep-frozen bull and boar spermatozoa when the diluent incorporates milk but a slight modification (see below) is required if a diluent has been used which contains egg yolk.

A mixture of $0.67 \mathrm{~g}$ eosin (yellowish, Gurr) and either $10 \mathrm{~g}$ nigrosin (referred to as nigrosin eosin-NE) or $5 \mathrm{~g}$ nigrosin (eosin nigrosin-EN) is added to $100 \mathrm{ml}$ tap water and brought to the boil. The stain is ready for use when it has cooled. Both stains remain stable indefinitely.

To prepare the smear, one drop of semen is added to at least eight drops of stain which has been heated to $30^{\circ} \mathrm{C}$. The mixture is incubated for $5 \mathrm{~min}$ at $30^{\circ} \mathrm{C}$ and smears made on preheated slides so that it is easy to see through the smear when it is held over a light background. The smear is then allowed to dry at $30^{\circ} \mathrm{C}$ and a portion of the smear covered, using either DPX or Eukitt as a mountant. 
At any time after the mountant has set (smears made 12 years ago have been treated successfully), the slide is shaken in a mixture of equal volumes of $5 \% \mathrm{v} / \mathrm{v}$ acetic acid and $0.6 \mathrm{~N}$-perchloric acid for 10 to $30 \mathrm{sec}$ and the excess is washed off in running tap water. The slide is immersed briefly in ethanol and then ether to remove the water, and another portion of the smear is then covered. For difficult material, such as semen diluted in an egg yolk diluent, the best results are obtained if the water used for washing is at $37^{\circ} \mathrm{C}$.

Good results can be obtained by substituting a $5 \%$ aqueous solution of aniline blue for the acetic acid/perchloric acid mixture. Prolonged immersion $(10 \mathrm{~min})$ in aniline blue, either instead of, or after the acetic acid/perchloric acid treatment (clearing), stains all the spermatozoa, the slide can then be rinsed briefly in tap water and dried as described above before covering.

After clearing, the eosinophilic spermatozoa are coloured red and are easily seen against a clear background (Plate 1), some of these spermatozoa have eosin associated only with the post-acrosomal region (Pl. 1, Figs. 1 inset ii, and 3 i) and in some others the acrosome has acquired a dark colouring (presumably from the nigrosin) which it retains even when it has become detached

\section{TABLE 1}

THE PERGENTAGE OF EOSINOPHILIC BULL SPERMATOZOA IN SMEARS STAINED EITHER WITH NIGROSIN EOSIN OR EOSIN NIGROSIN

\begin{tabular}{c|c|c|c}
\hline Stain & Uncleared & Cleared & Mean \\
\hline NE & 17.76 & 27.4 & 22.58 \\
EN & 40.07 & 41.88 & 40.97 \\
& 28.91 & 34.63 & \\
& & & \\
\hline
\end{tabular}

Values are shown for 'cleared' and 'uncleared' portions of the smears. Each figure is the mean of two counts of 100 spermatozoa on forty-nine slides. For abbreviations, see text.

from the spermatozoa (Pl. 1, Figs. 2 vi and 3 vii). Free acrosomes are not visible on the uncleared portion of the smear. A number of cytoplasmic droplets can also be seen on the cleared portion of the smear (Pl. 1, Fig. 1 and inset i). Non-eosinophilic spermatozoa are not easily seen on the cleared portion of the smear if it is viewed by bright-field transmitted light, but they appear nearly as dark as the eosinophilic spermatozoa when phase contrast is used. It is hoped this will be the basis of a method for obtaining counts of live/dead spermatozoa with an image-analysing computer.

Smears were made of bull spermatozoa taken from the epididymis, diluted with seminal plasma or various fractions of seminal plasma and subjected to temperature shock. Detailed results of this experiment will be published elsewhere. Half of these smears were stained with NE and half with EN (forty-nine of each). All the smears were cleared and counts of eosinophilic and noneosinophilic spermatozoa were made on cleared and uncleared portions of each smear. The mean values are presented in Table 1. 


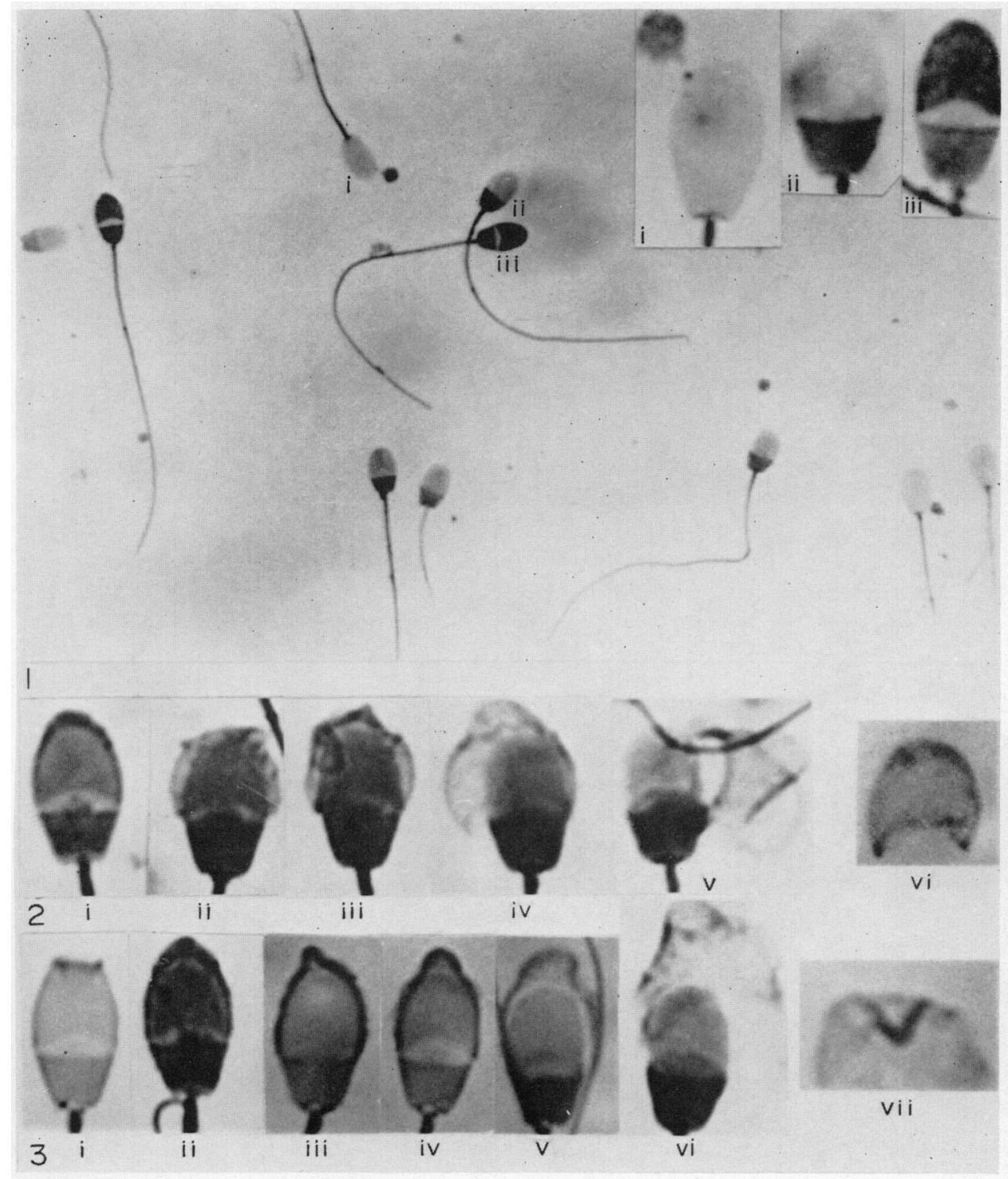

Ram spermatozoa stained with eosin nigrosin and 'cleared' (see text for method). Bright-field transmitted light.

FIG. 1. $\times 650$. (i) Non-eosinophilic spermatozoon, $\times 2000$. (ii) Spermatozoon stained in the post-acrosomal region only with eosin, $\times 2000$. (iii) Spermatozoon stained in the post-acrosomal region with eosin. The acrosome is stained with nigrosin, $\times 2000$.

Fig. 2. (i) to (v) Presumed successive stages in loss of the acrosome. (vi) 'Free' acrosome, $\times 2000$.

Fig. 3. (i) to (vi) Presumed successive stages in the loss of the acrosome from a 'knobbed' spermatozoa. (vii) 'Free' acrosome from a 'knobbed' spermatozoon. $\times 2000$.

(Facing p. 444) 
Analysis of variance confirms that fewer spermatozoa stained in NE than in EN $(P<0.01)$ and fewer stained spermatozoa were detected before clearing than after clearing $(P<0 \cdot 1)$. This applies particularly to smears stained with NE $(P<0 \cdot 5)$.

One explanation of the difference between the percentage of stained spermatozoa on cleared and uncleared slides might have been that some unstained spermatozoa are removed during the clearing process. To examine this possibility, six smears were made and selected fields were photographed before and after clearing: only twelve out of 267 spermatozoa were lost $(<5 \%)$. Even if all these spermatozoa had been non-eosinophilic, such a loss would only have increased the figure of $28.9 \%$ stained (Table 1) to approximately $30 \%$. It seems more likely that the discrepancy between NE and EN, and between cleared and uncleared NE smears can be accounted for by the presence of partially stained spermatozoa (Campbell, Dott \& Glover, 1956; Dott, 1956). Two observations which tend to confirm this are (1) it was observed that spermatozoa with eosin in the post-acrosomal region were easily detected in EN and in cleared smears of both stains but in cleared smears of NE (and to a lesser extent in uncleared NE smears) a slight darkening was observed in some spermatozoa which did not apparently have any eosin associated with them, and (2) on cleared slides, in counts of spermatozoa with eosin in the postacrosomal region only, there was a higher percentage of such spermatozoa in the EN smears.

The differentiation of a population of spermatozoa into eosinophilic and non-eosinophilic provides an indication of a difference in the membranes of individuals comprising the two groups. It is generally assumed that eosinophilic spermatozoa are those that have 'passed their best' because populations with a high proportion of these spermatozoa also have characteristics consistent with the presence of a high proportion of physiologically ineffective spermatozoa (Bishop, Campbell, Hancock \& Walton, 1954).

However, it is clear that the actual proportion of eosinophilic spermatozoa in a population is dependent, in part, on the method of estimation. It is therefore important that both the method of preparing the smears and the criteria used for assigning spermatozoa to the 'stained' or 'unstained' category should be rigidly applied, since only under these circumstances can valid comparisons be made between different samples of semen.

It is possible that more information on the process of sperm degeneration will be provided by the examination of spermatozoa that have been subjected to various stresses (such as freezing), stained with eosin and nigrosin and then cleared. It could be particularly useful for the examination of semen, samples of which are to be studied under the electron microscope.

\section{REFERENCES}

Bishop, M. W. H., Campbell, R. C., Hancock, J. \& Walton, A. (1954) Semen characteristics and fertility in the bull. F. agric. Sci., Camb. 44, 227.

CAMpbelt, R. C., DotT, H. M. \& Glover, T. D. (1965) Nigrosin eosin as a stain for differentiating live and dead spermatozoa. F. agric. Sci., Camb. 48, 1.

Dort, H. M. (1956) Partial staining of spermatozoa in the nigrosin eosin stain. Proc. 3rd Int. Cong. Anim. Reprod. A.I., Cambridge, A.I. Section, p. 42. 